\title{
Effects of external focus of attention on learning static balance among girls with ADHD
}

\author{
Saeed Ghorbani ${ }^{1}$, Amir Dana $^{2}$, Efstathios Christodoulides ${ }^{3}$ \\ 1 Department of Physical Education and Sport Sciences, Aliabad Katoul Branch, Islamic Azad University, Aliabad Katoul, \\ Iran; ${ }^{2}$ Department of Physical Education and Sport Sciences, Gonbad Kavoos Branch, Islamic Azad University, Gonbad \\ Kavoos, Iran; ${ }^{3}$ University of Central Lancashire, Cyprus
}

\section{Summary}

Study aim: The purpose of this study was to examine the effects of adopting an external focus of attention on motor learning among girls with ADHD aged seven and eleven years.

Material and methods: Twenty-four seven-year-old and 24 eleven-year-old female children with symptoms of ADHD were randomly assigned to groups receiving either external focus of attention (EXT) instructions or internal focus of attention (INT) instructions, making four experimental groups: EXT-7, INT-7, EXT-11, and INT-11. Participants performed a pretest followed by five training blocks under an external or internal instruction and were then given a retention test one day later. After training, we employed a manipulation check to verify the children's type and intensity of focus.

Results: Adopting an external focus of attention, compared to an internal focus of attention, led to better motor learning among girls with $\operatorname{ADHD}\left(F_{1,44}=5.08, p=0.029, \eta^{2}=0.10\right)$. In addition, adopting an external focus of attention reduced the children's tendency to focus on self. Older children performed better than younger children in balance time $\left(F_{1,44}=16.10, p<0.001\right.$, $\left.\eta^{2}=0.26\right)$.

Conclusions: Our results indicate that propositions of the OPTIMAL theory can be extended to children with ADHD.

Keywords: External focus of attention - ADHD - Motor learning - Static balance

\section{Introduction}

Attention deficit hyperactivity disorder (ADHD) is one of the most common neurodevelopment disorders in children, persisting long into adolescence and adulthood. It has been well established that children with ADHD show cognitive difficulties in inattentiveness, impulsivity, and hyperactivity [6]. Moreover, some studies have reported that children with ADHD typically show difficulties in performing and learning motor skills $[4,17]$. Considering the problems children with ADHD encounter performing and learning motor skills, finding and addressing suitable training factors and conditions for these children can positively influence their motor skill performance and is of great importance to them, their families, their physical educators and their occupational therapists.

Available research suggests that one of the most effective factors for optimizing motor learning is an external focus of attention [21, 22, 24, 25]. Adopting an external focus of attention is considered as an important factor in the OPTIMAL (Optimizing Performance through Intrinsic Motivation and Attention for Learning) theory of motor learning [24]. Based on the OPTIMAL theory, practice conditions that facilitate motor performance and learning are (a) enhanced expectancies, (b) autonomy support and (c) external focus of attention. In this theory, enhanced expectancies and autonomy support are considered as motivational factors and have been well investigated by previous research [7, 9, 13, 14]. In addition, external focus of attention is considered as an attentional factor, which optimizes performance and learning a new motor skill [24].

Several studies have examined the impact of different strategies for focusing attention on motor skill learning $[1-3,21-23,26,27]$. These studies generally compared the effects of adopting an external versus internal focus of attention for this purpose. An external focus of attention refers to the focusing on the intended environmental movement such as the dartboard or path of the dart in dart throwing, while an internal focus of attention refers to 
focusing on the body such as the hand during dart throwing [24].

A common finding in previous research was that adopting an external focus of attention had a better performance and motor learning impact than adopting an internal focus of attention. For instance, Wulf and Su [27] asked novices to hit golf balls into a circular target and found that participants instructed to focus internally on the swinging motion of their arms, versus those who focused externally on the pendulum-like motion of the club, had significantly lower accuracy scores on the learning retention test. In addition, Wulf et al. [23] replicated these results by using a soccerthrowing task with children. Moreover, Abdollahipour et al. [2] found that children who adopted an external (versus internal) focus of attention showed greater bowling accuracy. Wulf and Lewthwaite [24] proposed that adopting an external focus of attention optimizes motor performance and facilitates motor learning by directing attention to the task goal and reducing the focus on the self. Thus, an external focus of attention is clearly an important contributor to goal-action coupling [24].

While most studies that have investigated the impact of focus of attention on performance and learning of motor skills involved typically developing people, only a few researchers have sought to determine whether these findings would hold for special populations. Saemi, Porter, Wulf, Ghotbi-Varzaneh, and Bakhtiari [20] investigated the impact of adopting an external (versus internal) focus of attention on performance and learning of throwing a tennis ball into a target in a group of children with ADHD. These children practiced six blocks of thirty trials during the acquisition phase and completed retention testing over 10 trials two days later. The results showed that adopting an external focus of attention resulted in better accuracy scores than adopting an internal focus of attention in both the acquisition and retention test. Chiviacowsky, Wulf, and Avila [8] further investigated this issue in children with intellectual disabilities by using a task similar to the one used in the study of Saemi et al. [20]. Children practiced four blocks of eight trials during acquisition followed by 10 trials of retention and transfer tests. These results did not find that an external focus of attention led to better accuracy scores during training, but an external focus was superior for accuracy scores in the retention and transfer tests. These authors suggested that adopting an external focus of attention led to a more autonomic, efficient, and accurate throwing pattern and reduced attentional demands for these children $[8,20]$.

Although these studies increased our knowledge of the effectiveness of an external focus of attention on performance and learning of motor skills in special groups, additional studies using different motor tasks are needed to confirm that these external focus advantages can be extended to special populations, perhaps particularly children with ADHD. Therefore, the aim of this study was to investigate the effects of an external versus internal focus of attention on performance and learning of a static balance task (an important motor skill in school-aged children) among children with ADHD. Based on the foregoing prior research, we hypothesized that adopting an external versus internal focus of attention would result in better motor learning and better retention performance. Further, because we were interested in any developmental differences regarding these learning factors among children with ADHD, we compared children with ADHD who were seven versus eleven-year-old. Here, we hypothesized that eleven-year-old children would show better motor performance than seven-year-old children.

\section{Material and methods}

\section{Participants}

Forty-eight female children attending special school for children with ADHD participated in this study. All children had symptoms of ADHD, and the two age groups were evenly distributed in this sample (24 seven-yearolds; 24 eleven-year-olds). Within each age group, participants were randomly assigned to groups receiving either external focus of attention (EXT) instructions or internal focus of attention (INT) instructions. Thus, these children were distributed across four different experimental groups: EXT-7, INT-7, EXT-11, and INT-11. The protocol was performed in accordance with the Declaration of Helsinki and approved by the university's institutional review board. Parents of all child participants gave their informed written consents for the children to participate.

\section{Motor task}

A static balance device (i.e., warrior III pose) was selected as the motor task in this study. This task requires the participant to balance on the right leg while lifting the left leg off the ground and keeping the arms over the head (see Fig. 1). The aim of the motor task in this study was to maintain this posture (Fig. 1) for as long as possible. We measured the length of each child's balance time with a digital stopwatch in minutes, starting the stopwatch when the child put her body into this posture and stopping it when she made a positional error such as hitting the ground with her hands or left leg.

\section{Procedure}

We completed a demographic data sheet for each child by referring to the child's school profile. Each child was tested individually in the school gym. Upon entering the gym, we gave the child general information about the study including details about the procedure and the motor task. To familiarize the children with the experimental setting and motor task, we asked participants to perform one trial within 


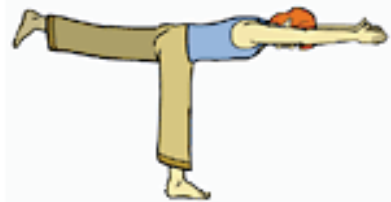

Fig. 1. Schematic view of the motor task

a marked area designated as the balancing area in this study. Participants then performed one trial as a pretest without any attentional focus instructions. Then, they engaged in the acquisition phase over five training blocks with each consisting of three minutes of training on the balance task. The participants were given a two-minute resting time between each training block. One day later, the children performed a one-trial retention test without any attentional focus instructions. In the pretest and the retention test, the children were instructed to balance as long as possible. In the external focus condition, the children were instructed to "focus on the yellow marker" that was placed two meters ahead of them on the ground. In the internal focus condition, the children were instructed to "focus on the right leg" while balancing. In the retention test, there were neither attentional focus instructions nor a yellow marker on the ground. To ensure that the children followed the focus instructions, we reminded them orally on how to focus at 10 -second intervals during the training block. In order to measure the type and intensity of focus the participants applied, we had all children perform a manipulation check following the training phase. To determine the type of focus, we asked "what did you focus on?", and to measure intensity of focus, we asked them to indicate on a Likert scale from 1 (not at all) to 7 (very much) "how much did you focus on it?"

\section{Data analysis}

Dependent measures were length of balance time (in minutes) during the pretest and retention test and their report of the type and intensity of focus (measured by manipulation check). We used a 2 (FOCUS: external vs. internal) $\times 2$ (AGE: seven vs. eleven) analysis of variance (ANOVA) to analyze time of balance during the pretest and retention test, and we analyzed intensity of focus with a one-way ANOVA. We set the statistical significance level at $p<0.05$.

\section{Results}

\section{Balance time}

In the pretest, the ANOVA showed a significant main effect for AGE, $F_{1,44}=13.48, p=0.001, \eta^{2}=0.23$, but there was no significant main effect for FOCUS, $F_{1,44}=0.76$, $p=0.386$. Moreover, the FOCUS $\times$ AGE interaction was not significant, $F_{1,44}=1.41, p=0.240$. As shown in Figure 2 , the balance time of eleven-year-old children was longer than that of seven-year-old children in the pretest. In the retention test, the ANOVA demonstrated a significant main effect for both AGE, $F_{1,44}=16.10, p<0.001$, $\eta^{2}=0.26$ and FOCUS, $F_{1,44}=5.08, p=0.029, \eta^{2}=0.10$, but the FOCUS $\times$ AGE interaction was not significant, $F_{1,44}=0.001, p=0.984$. Figure 2 also shows for the retention test that the balance time of eleven-year-old children was longer than that of seven-years-old children. In addition, in the retention test the children in EXT groups had longer balance time than those in INT groups indicating that EXT groups performed significantly better than INT groups.

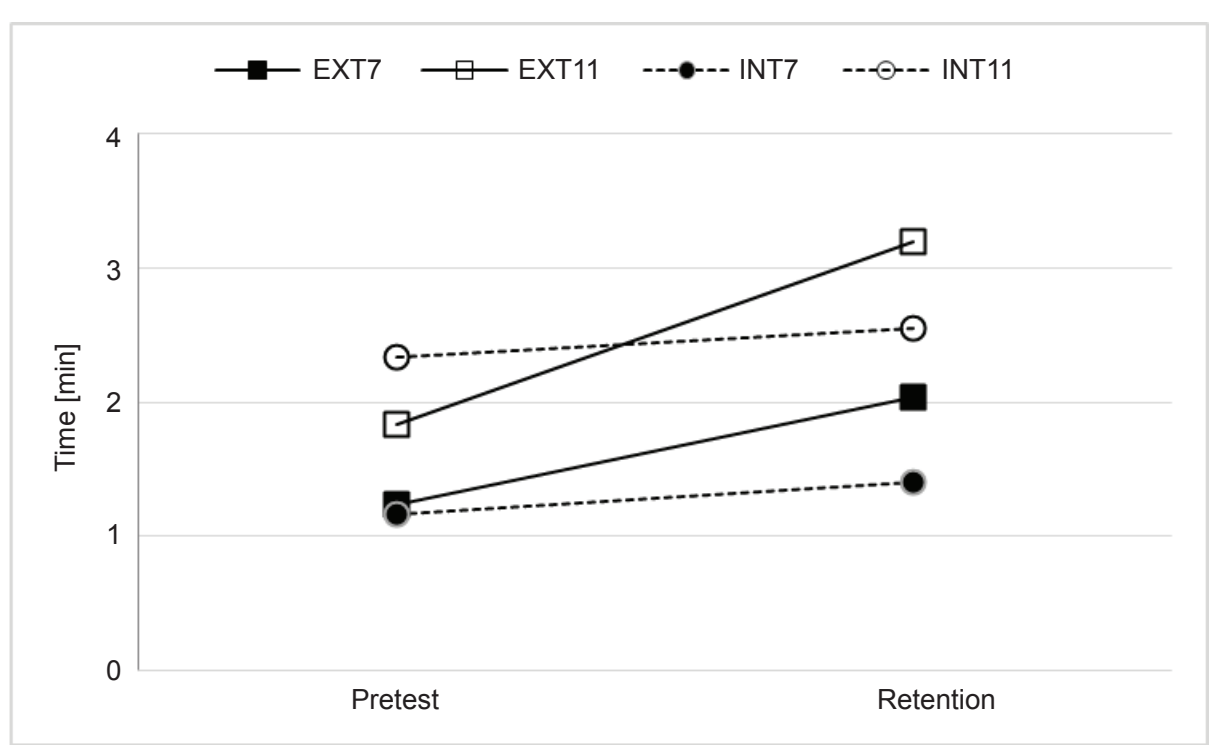

Fig. 2. Time of balance for the experimental groups across pretest and retention test (EXT: external focus; INT: internal focus) 
Table 1. Participants' responses to the manipulation check

\begin{tabular}{llcccc}
\hline & & \multicolumn{2}{c}{ External focus reported } & \multicolumn{2}{c}{ Internal focus reported } \\
\hline & \multicolumn{2}{c}{ Yellow marker } & \multicolumn{2}{c}{ Body } \\
\hline \multirow{2}{*}{ EXT } & What & $83.3 \%$ & $91.7 \%$ & $16.7 \%$ & $8.3 \%$ \\
& Intensity & 5.1 & 5.1 & 5.5 & 5 \\
\multirow{2}{*}{ INT } & $33.3 \%$ & $16.6 \%$ & $67.7 \%$ & $83.3 \%$ \\
& What & 5.5 & 6 & 5.3 & 5.2 \\
& Intensity & & &
\end{tabular}

* EXT: external focus; INT: internal focus

\section{Manipulation check}

Our manipulation check revealed that children who adopted an external focus of attention reported that they focused generally on the yellow marker and children who adopted an internal focus reported that they focused generally on their body (see Table 1). There were no significant group differences in the reported intensity of participants' focus, $F_{3,44}=0.33, p=0.803$.

\section{Discussion}

Among special populations of participants, the effects of adopting an external focus of attention have rarely been investigated. In this study, we used a balance task to determine whether a generally advantageous external focus of attention would assist motor learning among girls with ADHD. As hypothesized, this study demonstrated that children who adopted an external focus of attention during balance training performed better in the subsequent retention test than children who adopted an internal focus of attention. This finding is in line with previous results that showed benefits for adopting an external focus of attention rather than adopting an internal focus of attention in learning new motor skills in both typically developing and in a few previously studied special groups [1-3, 8, 12, 20, 22, 23, 26, 27]. In addition, this finding provided support for the OPTIMAL theory of motor learning [24] that postulated an advantage for adopting an external focus of attention when learning new motor skills.

Importantly, we confirmed through the childrens' reports that those who adopted an external focus of attention focused mostly on an external cue (yellow marker) rather than an internal cue (body) while performing the motor task, whereas the reverse was true for children who adopted an internal focus of attention. According to the OPTIMAL theory, adopting an external focus of attention facilitates motor learning by directing attention to the task goal and reducing the focus on the self [24]. The findings of this study provide support for this proposition by showing that adopting an external focus of attention promotes focusing on task goal and reduces focusing on the self. Thus, an external focus of attention is an important contributor to goal-action coupling. This may be particularly important for children with ADHD who are subject to distractions.

Regarding age differences among the girls with ADHD in our study, we found that older children (eleven-yearolds) outperformed younger children (seven-year-olds) in length of balancing time. This finding is in accordance with Flores et al. [12], who found that ten-year-old typically developing children performed significantly better than sixyear-old typically developing children in the transfer test of a dynamic balance task. This finding is not surprising, as previous studies have demonstrated that older children show better motor coordination and more advanced development generally than do younger children $[10,19]$. Similarly, older children with ADHD are considered to have fewer motor coordination problems than younger children with ADHD [11]. However, our findings add to this literature by specifying that the superior performance of older children extends to older and younger girls with ADHD on a static balance task. Of note, however, we found no interaction between AGE and FOCUS in the retention test, suggesting that both age groups of girls with ADHD benefitted from an external focus of attention as the OPTIMAL theory would predict.

Among the limitations of the present study is the fact that we used only girls as participants, perhaps limiting the study's generalizability to males until further research with both genders can replicate these findings. Similarly, our restricted age groups of girls aged seven and eleven years raises questions about whether these results would extend to older or younger children, and this should be addressed by future researchers. Also, 
we examined the effects of focus of attention on balance time but did not use a motor learning task in which movement coordination was measured. Future studies should investigate the effects of different focus of attention on movement coordination among children with ADHD with emphasis on kinematic analysis [5, 15] and might utilize tasks that require a longer period of sustained attention, known to be a weakness for this group. Finally, we suggest that future studies should examine the effects of other therapy programs in combination with stimulation of sensomotoric development on improving postural stability deficits in children with postural problems [18].

Conflict of interest: Authors state no conflict of interest.

\section{References}

1. Abdollahipour R., Land W.M., Cereser A., Chiviacoesky S. (2019) External relative to internal attentional focus enhances motor performance and learning in visually impaired individuals. Disabil. Rehabil., 8: 1-10.

2. Abdollahipour R., Nieto M.P., Psotta R., Wulf G. (2017) External focus of attention and autonomy support have additive benefits for motor performance in children. Psychol. Sport Exerc., 32: 17-24.

3. Abdollahipour R., Wulf G., Psotta R., Nieto M.P. (2015) Performance of gymnastics skill benefits from an external focus of attention. J. Sports Sci., 33: 1807-1813.

4. Barnes K.A., Howard J.H Jr., Howard D.V., Kenealy L., Vaidya C.J. (2010) Two forms of implicit learning in childhood ADHD. Dev. Neuropsychol., 35: 494-505.

5. Bund A., Ghorbani S., Rathjens F. (2016) A three-dimensional movement analysis of the spike in Fistball. Sports, 4(4): 55.

6. Capodieci A., Lachina S., Cornoldi C. (2018) Handwriting difficulties in children with attention deficit hyperactivity disorder (ADHD). Res. Dev. Disabil.. 74: 41-49.

7. Chiviacowsky S. (2014) Self-controlled practice: Autonomy protects perceptions of competence and enhances motor learning. Psychol. Sport. Exerc., 15: 505-510.

8. Chiviacowsky S., Wulf G., Avila L.T.G. (2010) An external focus of attention enhances motor learning in children with intellectual disabilities. J. Intellect. Disabil. Res., 57(7): 627-634.

9. Chiviacowsky S., Wulf G., Lewthwaite R. (2012) Selfcontrolled learning: the importance of protecting perceptions of competence. Front. Psychol., 3, Article 458.

10. de Chaves R.N., Bustamante Valdívia A., Nevill A., Freitas D., Tani G., Katzmarzyk P.T., Maia J.A. (2016) Developmental and physical-fitness associations with gross motor coordination problems in Peruvian children. Res. Dev. Disabil., 53-54: 107-114.

11. Fliers E., Rommelse N., Vermeulen S.H., Altink M., Buschgens C.J., Faraone S.V., Sergeant J.A., Franke B., Buitelaar J.K. (2008). Motor coordination problems in children and adolescents with ADHD rated by parents and teachers: Effects of age and gender. J. Neural Transm., 115(2): 211-220.

12. Flores F.S., Gomes Schild J.F., Chiviacowsky S. (2015) Benefits of external focus instructions on the learning of a balance task in children of different ages. Int. J. Sport Psychol., 46(4): 311-320.

13. Ghorbani S. (2019) Motivational effects of enhancing expectancies and autonomy for motor learning: An examination of the OPTIMAL theory. J. Gen. Psychol., 146(1): 79-92.

14. Ghorbani S., Bund A. (2020) Motivational effects of enhanced expectancies for motor learning in individuals with high and low self-efficacy. Percept. Mot. Skill., 127(1): 263-274.

15. Ghorbani S., Bund A. (2017) Throwing skills: Analysis of movement phases in early motor learning. Percept. Mot. Skill., 124(2): 502-513.

16. Ghorbani S., Dana A., Fallah Z. (2019) The effects of external and internal focus of attention on motor learning and promoting learner's focus. Biomed. Hum. Kinet., 11: 175-180.

17. Goulardins J.B., Marques J.C.B., De Oliveira J.A. (2017) Attention Deficit Hyperactivity Disorder and Motor Impairment: A Critical Review. Percept. Mot. Skill., 124(2): 425-440.

18. Maciaszek J., Kilan N., Bronikowski M. (2016) Reaction to the sensory integration therapy in children with postural stability deficits. Minerv. Pediatr., 27706121.

19. Magalhaes L.C., Koomar J.A., Cermak S.A. (1989) Bilateral motor coordination in 5- to 9-year-old children: A pilot study. Am. J. Occup. Ther., 43(7): 437-443.

20. Saemi E., Porter J.M., Wulf G., Ghotbi-Varzaneh A., Bakhtiari S. (2013) Adopting an external focus of attention facilitates motor learning in children with attention deficit hyperactivity disorder. Kinesiology, 45, 179-185.

21. Wulf G. (2007) Attention and motor skill learning. Champaign: Human Kinetics.

22. Wulf G. (2013) Attentional focus and motor learning: A review of 15 years. Int. Rev. Sport Exerc. Psychol., 6: 77-104.

23. Wulf G., Chiviacowsky S., Schiller E., Ávila L.T. (2010) Frequent external-focus feedback enhances learning. Front. Psychol., 1: 190.

24. Wulf G., Lewthwaite R. (2016) Optimizing performance through intrinsic motivation and attention for learning: the OPTIMAL theory of motor learning. Psychon. Bull. Rev., 23: 1382-1414. 
25. Wulf G., Lewthwaite R. (2017) Optimizing motivation and attention for motor performance and learning. Curr. Opin. Psychol., 16: 38-42.

26. Wulf G., Lewthwaite R., Cardozo P., Chiviacowsky S. (2018) Triple play: Additive contributions of enhanced expectancies, autonomy support, and external attentional focus to motor learning. Q. J. Exp. Psychol., 71(4): 824-831.

27. Wulf G., Su J. (2007) External focus of attention enhances golf shot accuracy in beginners and experts. Res. $Q$. Exercise Sport, 78: 384-389.
Received 16.12.2019

Accepted 15.02.2020

\section{(C) University of Physical Education, Warsaw, Poland}

\section{Acknowledgments}

We thank all children and their parents and teachers for participating in this study. 